\title{
STABILITY ABOUT LIBRATION POINTS FOR RESTRICTED FOUR-BODY PROBLEM
}

\author{
Ismail. M.N. ${ }^{1 *}$, Ibrahim.A.H ${ }^{1}$, Zaghrout.A.S ${ }^{2}$, Younis, S.H ${ }^{2}$, Elmalky.F.S ${ }^{2}$, El-Masry.L.E ${ }^{3}$. \\ ${ }^{1}$ Astronomy and Meteorology Department, Faculty of Science, Al-Azhar University, Cairo, Egypt. \\ ${ }^{2}$ Mathematics Department, Faculty of Science (Girls), Al-Azhar University, Cairo, Egypt. \\ ${ }^{3}$ Ph.D student in Math. Dep., Faculty of Science (Girls), Al-Azhar University, Cairo.
}

*Corresponding author mail: mnader_is@azhar.edu.eg

\begin{abstract}
In this work, the Restricted Four-Body Problem is formulated in Hamiltonian form. The canonical form for the system is obtained which represents the equations of motion. The collinear libration points are obtained, we have five collinear libration points. The non-collinear libration points are found which are three non collinear libration points, they are obtained for different angles between the sight of Sun and the plane of Earth-Moon. The periodic orbits around each of these libration points are studied using two methods. The first method depends on the reduction of order of differential equations and the second method depends on the Eigen values of the characteristic equation. Two codes of MATHEMATICA are constructed to apply these two methods on the Sun- Earth-Moon-Spacecraft. The Poincare sections are obtained using the first method, these sections are used to illustrate the intersect points of the trajectories with the plane perpendicular to the plane of motion about each of the collinear libration points. Mirror symmetry is explored about each of these points. The Lyapunov orbits, and the Lissajous orbits about each of the collinear libration points are the results obtained by the second method. The eccentricities and the periods of each orbit are obtained. This study illustrates that the motion about the libration point L2 is more stable than the motion about any other collinear libration points.
\end{abstract}

Keywords: Lissajous orbits, Poincare surface sections, Libration points, Stability of libration points, restricted four-body, motion near libration points.

\section{INTRODUCTION}

The four-body problem has been extensively studied in astrodynamics. There are several attempts to model the four-body problem in mathematical form, which has been studied by many authors; for example, the bicircular model for the Earth-Moon-Sun system is studied by [1-2-3] in which the Earth and the Moon are considered as two primaries revolving in circular orbits around their barycenter, while the Sun is considered as a perturbing body moving in a circular orbit around the central masses of the Earth-MoonSun system. The Sun, Earth, and Moon are treated as distinct particles of finite mass [4-56]. The quasi-bi-circular model, which made an investigation into the phase space in the vicinity of the Sun-perturbed Earth-Moon collinear equilibrium points is used by [7]. The restricted Hill four-body model and determination periodic motion in the vicinity of the Sun-perturbed Earth-Moon triangular points
(L4 and L5) are studied by [8]. The invariant manifolds associated with these solutions were also examined. We study the restricted fourbody problem as equal masses located at the vertices of an equilateral triangle which investigated the equilibrium points, zero velocity curves, and families of periodic orbits were studied by [9-10-11-12]. The stability, periodic orbits around the triangular points of the Earth-Moon system, (which is perturbed by the Sun), and calculation of a periodic solution for the Sun-Earth-Moon system using numerical integration is proved by [13-14]. The numerical technique of Poincare surface sections is used to generate periodic and quasiperiodic orbits $[15,16]$. The Lissajous orbits and Lyapunov orbits around the collinear points in the restricted three-body problem under the effect of oblateness is found by [17]. In this paper, the canonical form is derived from the Hamiltonian formulation for the restricted fourbody problem. The libration points are obtained, and the stability of motion about each 
ISMAIL. M.N., et al.,

collinear libration points is studied. The Poincare surface sections are applied to the Sun-Earth-Moon-spacecraft system to illustrate the stability of motion about each libration point.

\section{Hamiltonian of the problem}

Let $(x, y, z)$ be the inertial frame with origin $\mathrm{O}$ at the center of mass of the primaries $\mathrm{m}_{1}$ and $\mathrm{m}_{2}$, $\mathrm{x}$-axis represent the position from $\mathrm{m}_{1}$ to $\mathrm{m}_{2}$, as shown in figure 1 . We'll introduce the problem formulation under the following restrictions:

1- The masses $m, m_{1}$ and $m_{2}$ are moving in one plane.

2- $m_{3}$ Considered as a perturbing body.

3- Since we are interested in the dependence of the motion of the infinitesimal mass $m$, then $m_{3} \gg m_{1} \gg m_{2} \gg m$, which doesn't affect essentially on the generality of the problem.

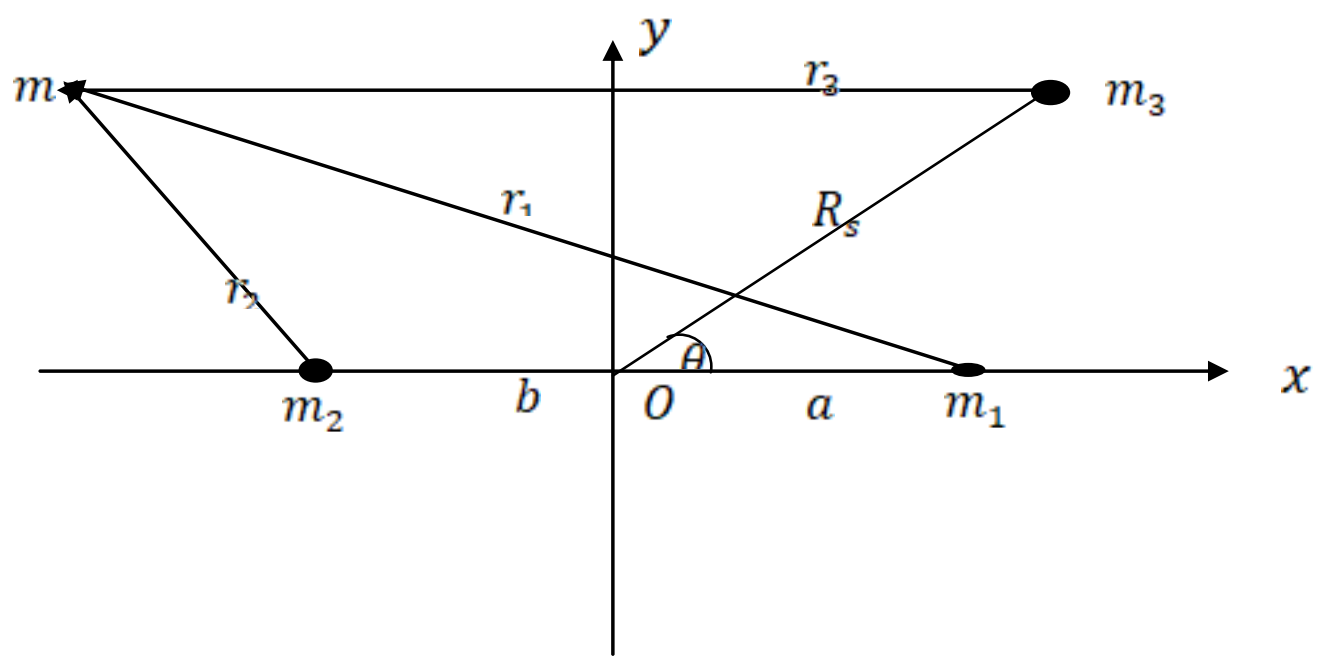

Fig. 1: The Restricted Four-Body Problem in an inertial frame.

As shown in figure 1 the coordinates of the masses are given by

For $m_{1}: x_{1}=a \quad, \quad y=0, \quad z_{1}=0$

For $m_{2}: x_{2}=b \quad, \quad y=0, \quad z_{2}=0$

For $m_{3}: \quad x_{3}=R_{s} \cos \theta, \quad y_{3}=R_{s} \sin \theta, \quad z_{3}=0$

where,

$$
\begin{aligned}
r_{1} & =\left[\left(x-x_{1}\right)^{2}+\left(y-y_{1}\right)^{2}+\left(z-z_{1}\right)^{2}\right]^{\frac{1}{2}} \\
& =\left[(x-a)^{2}+(y)^{2}+(z)^{2}\right]^{\frac{1}{2}} \\
r_{2} & =\left[(x-b)^{2}+(y)^{2}+(z)^{2}\right]^{\frac{1}{2}} \\
r_{3} & =\left[\left(x-R_{s} \cos \theta\right)^{2}+\left(y-R_{s} \sin \theta\right)^{2}+(z)^{2}\right]^{\frac{1}{2}}
\end{aligned}
$$

$R_{s}$ : The distance between the $\mathrm{m}_{3}$ and the center of mass of the system.

$\theta$ : The angle between the sight of the $\mathrm{m}_{3}$ and the plane of Earth-Moon.

$$
\begin{aligned}
& a=\frac{m_{2} l}{m_{1}+m_{2}} \\
& b=\frac{m_{1} l}{m_{1}+m_{2}}
\end{aligned}
$$


Such that $l$ is the total distance between $m_{1}$ and $m_{2}$.

$$
l=a+b
$$

Applying the concept of Lagrange and Hamiltonian principle in rotating frame on the restricted fourbody problem [20], then we have

$L(x, y, z, \dot{x}, \dot{y}, \dot{z})=T-U=\frac{1}{2}\left[(\dot{x}-y)^{2}+(\dot{y}+x)^{2}+\dot{z}^{2}\right]+G\left[\frac{m_{1}}{r_{1}}+\frac{m_{2}}{r_{2}}+\frac{m_{3}}{r_{3}}\right]$

After some few algebraic reductions

$$
\begin{gathered}
L(x, y, z, \dot{x}, \dot{y}, \dot{z})=\frac{1}{2}\left[\left(\dot{x}^{2}+\dot{y}^{2}+\dot{z}^{2}\right)+\left(x^{2}+y^{2}\right)+2(x \dot{y}-\dot{x} y)\right]+ \\
G\left[\frac{m_{1}}{r_{1}}+\frac{m_{2}}{r_{2}}+\frac{m_{3}}{r_{3}}\right]
\end{gathered}
$$

By using the dimensionless coordinates, then equation (5) will be

$$
L(x, y, z, \dot{x}, \dot{y}, \dot{z})=\frac{1}{2}\left[\left(\dot{x}^{2}+\dot{y}^{2}+\dot{z}^{2}\right)+\left(x^{2}+y^{2}\right)+2(x \dot{y}-\dot{x} y)\right]+\left[\frac{1-\mu}{r_{1}}+\frac{\mu}{r_{2}}+\frac{\mu_{s}}{r_{3}}\right]
$$

Where, $\mu=\frac{m_{2}}{m_{1}+m_{2}}, 1-\mu=\frac{m_{1}}{m_{1}+m_{2}}, \mu_{s}=\frac{m_{3}}{m_{1}+m_{2}}[21]$

By using the definition of the momentum $p_{i}=\frac{\partial L}{\partial \dot{q}_{i}}$, this yields

$$
\begin{aligned}
& p_{x}=\frac{\partial L}{\partial \dot{x}}=(\dot{x}-y) \\
& p_{y}=\frac{\partial L}{\partial \dot{y}}=(\dot{y}+x) \\
& p_{z}=\frac{\partial L}{\partial \dot{z}}=\dot{z}
\end{aligned}
$$

From Eqns. (7), it is clear that

$$
\begin{aligned}
& \dot{x}=p_{x}+y \\
& \dot{y}=p_{y}-x \\
& \dot{z}=p_{z}
\end{aligned}
$$

The Hamiltonian is defined [20]

$$
\begin{aligned}
\mathcal{H}= & \sum_{i} p_{i} \dot{q}_{i}-L \\
\mathcal{H}= & \left(p_{x} \dot{x}+p_{y} \dot{y}+p_{z} \dot{z}\right)-L \\
\mathcal{H}= & \left(p_{x} \dot{x}+p_{y} \dot{y}+p_{z} \dot{z}\right)-\frac{1}{2}\left[\left(\dot{x}^{2}+\dot{y}^{2}+\dot{z}^{2}\right)+\left(x^{2}+y^{2}\right)+2\left(x \dot{y}-\dot{x}_{s}\right.\right. \\
& {\left[\frac{1-\mu}{r_{1}}+\frac{\mu}{r_{z}}+\frac{\mu_{s}}{r_{3}}\right] }
\end{aligned}
$$

Substitute from Eqns. (8) into Eq. (11), the Hamiltonian of the restricted four-body problem is given by

$$
\mathcal{H}=\frac{1}{2}\left(p_{x}^{2}+p_{y}^{2}+p_{z}^{2}\right)+y p_{x}-x p_{y}-\left[\frac{1-\mu}{r_{1}}+\frac{\mu}{r_{2}}+\frac{\mu_{s}}{r_{3}}\right]
$$




\subsection{Canonical Form.}

The canonical form of the Hamiltonian are derived as

$$
\begin{aligned}
& \dot{x}=\frac{\partial \mathcal{H}}{\partial p_{x}}=p_{x}+y \\
& \dot{y}=\frac{\partial \mathcal{H}}{\partial p_{y}}=p_{y}-x \\
& \dot{z}=\frac{\partial \mathscr{H}}{\partial p_{z}}=p_{z} \\
& \dot{p}_{x}=-\frac{\partial \mathscr{H}}{\partial x}=p_{y}-\frac{(1-\mu)(x+\mu)}{\left((x+\mu)^{2}+y^{2}+z^{2}\right)^{\frac{2}{2}}}-\frac{\mu(x+\mu-1)}{\left((x+\mu-1)^{2}+y^{2}+z^{2}\right)^{\frac{2}{2}}}-\frac{\mu_{s}(x-\mathrm{Rs} \cos \theta)}{\left((x-\mathrm{Rs} \cos \theta)^{2}+(y-\mathrm{Rs} \sin \theta)^{2}+z^{2}\right)^{\frac{2}{2}}} \\
& \dot{p}_{y}=-\frac{\partial \mathscr{F}}{\partial y}=-p_{x}-\frac{(1-\mu) y}{\left((x+\mu)^{2}+y^{2}+z^{2}\right)^{\frac{2}{2}}}-\frac{\mu y}{\left((x+\mu-1)^{2}+y^{2}+z^{2}\right)^{\frac{2}{2}}}-\frac{\mu_{g}(y-\mathrm{R} \sin \theta)}{\left((x-\mathrm{R} \sin \cos \theta)^{2}+(y-\mathrm{R} \sin \theta)^{2}+z^{2}\right)^{\frac{2}{2}}} \\
& \dot{p}_{z}=-\frac{\partial \mathscr{H}}{\partial z}=-\frac{(1-\mu) z}{\left((x+\mu)^{2}+y^{2}+z^{2}\right)^{\frac{3}{2}}}-\frac{\mu z}{\left((x+\mu-1)^{2}+y^{2}+z^{2}\right)^{\frac{3}{2}}}-\frac{\mu_{s} z}{\left((x-\text { Rs } \cos \theta)^{2}+(y-\text { Rs } \sin \theta)^{2}+z^{2}\right)^{\frac{3}{2}}}
\end{aligned}
$$

Equations (13) are represented the equations of motion of the fourth body under effects of the gravitational forces of the three other primaries.

\subsection{The collinear libration points}

To obtain the collinear libration points, substituting in equations (13) by $\dot{x}=0, \dot{y}=0, \dot{z}=0, \dot{p}_{x}=0, \dot{p}_{y}=0, \dot{p}_{z}=0, y=0$ and $z=0$

Then, from eqns. (13.2) and (13.4)

$$
x-\frac{(1-\mu)}{(x+\mu)^{2}}-\frac{\mu}{(x+\mu-1)^{2}}-\frac{\mu_{S}(x-\mathrm{Rs} \cos \theta)}{\left((x-\mathrm{Rs} \cos \theta)^{2}+(-\mathrm{Rs} \sin \theta)^{2}\right)^{\frac{3}{2}}}=0
$$

If $\theta=0$., The collinear libration points, equation (14) becomes

$$
x-\frac{(1-\mu)}{(x+\mu)^{2}}-\frac{\mu}{(x+\mu-1)^{2}}-\frac{\mu_{s}}{(x-\mathrm{Rs})^{2}}=0
$$

Let the x-coordinates of the libration points, $x=x_{L}$ in Eq. (15) and then the equation up to fifth order of $x_{L}$ we have

$x_{L}^{5}\left(R s^{3}-\mu_{s}\right)+x_{L}^{4}\left(-2 R s^{3}+4 R s^{3} \mu+2 \mu_{s}+R s \mu_{s}-4 \mu \mu_{s}\right)+x_{L}^{3}\left(R s^{3}-6 R s^{3} \mu+\right.$

$\left.6 R s^{3} \mu^{2}-\mu_{s}-2 R s \mu_{s}+6 \mu \mu_{s}+4 R s \mu \mu_{s}-6 \mu^{2} \mu_{s}\right)+x_{L}^{2}\left(-R s^{3}+2 R s^{3} \mu-6 R s^{3} \mu^{2}+\right.$

$\left.4 R s^{3} \mu^{3}+R s \mu_{s}-2 \mu \mu_{s}-6 R s \mu \mu_{s}+6 \mu^{2} \mu_{s}+6 R s \mu^{2} \mu_{s}-4 \mu^{3} \mu_{s}\right)+x_{L}\left(2 R s^{3}-\right.$

$4 R s^{3} \mu+R s^{3} \mu^{2}-2 R s^{3} \mu^{3}+R s^{3} \mu^{4}+2 R s \mu \mu_{s}-\mu^{2} \mu_{s}-6 R s \mu^{2} \mu_{s}+2 \mu^{3} \mu s+$

$\left.4 R s \mu^{3} \mu_{s}-\mu^{4} \mu s\right)+3 R s^{3} \mu-3 R s^{3} \mu^{2}+R s \mu^{2} \mu_{s}-2 R s \mu^{3} \mu_{s}+R s \mu^{4} \mu_{s}-R s^{3}=0$

Equation (16) is equation of fifth order in $x_{L}$ and its solution has five real roots, each root specifies the position of each of the collinear libration points and it depends on the parameters $\mu_{s y} \mu$ and $R s$.

\subsection{The non-collinear libration points.}

Similarly, the conditions to obtain the non-collinear points libration points are $\dot{x}=0, \dot{y}=0, \dot{p}_{x}=0, \dot{p}_{y}=0$ and $z=0$ by substituting into Eqns.(13.1),(13.2),(13.4) and (13.5) then,

$$
\begin{aligned}
& x-\frac{(1-\mu)(x-\mu)}{\left((x-\mu)^{2}+y^{2}\right)^{\frac{3}{2}}}-\frac{\mu(x+\mu-1)}{\left((x+\mu-1)^{2}+y^{2}\right)^{\frac{3}{2}}}-\frac{\mu_{S}\left(x-R_{S} \cos \theta\right)}{\left(\left(x-R_{S} \cos \theta\right)^{2}+\left(y-R_{S} \sin \theta\right)^{2}\right)^{\frac{3}{2}}}=0 \\
& y-\frac{(1-\mu) y}{\left((x-\mu)^{2}+y^{2}\right)^{\frac{3}{2}}}-\frac{\mu y}{\left((x+\mu-1)^{2}+y^{2}\right)^{\frac{3}{2}}}-\frac{\mu_{S}\left(y-R_{S} \sin \theta\right)}{\left(\left(x-R_{S} \cos \theta\right)^{2}+\left(y-R_{S} \sin \theta\right)^{2}\right)^{\frac{3}{2}}}=0
\end{aligned}
$$


The non -collinear points are obtained by solving Eqns. (17) with different values of $\theta$, in this case, the non-collinear libration points vary with $\theta$.

\section{Jacobi Integral}

The potential for the system is

$$
U=\frac{1-\mu}{r_{1}}+\frac{\mu}{r_{2}}+\frac{\mu_{S}}{r_{3}}+\frac{x^{2}+y^{2}}{2}
$$

The Jacobi integral is obtained from [18]

$p_{x}{ }^{2}+p_{y}{ }^{2}+p_{z}{ }^{2}=2 U-C$

This equation is called the Jacobi integral, when velocity equals to zero, equation (18) becomes

$$
2 U=C
$$

Which enables to obtain the zero velocity curves and is used to study the Poincare surface sections (PSS). Table (1) displays the five collinear libration points and the Jacobi constant for each collinear libration points in the Sun-Earth-Moon system. Figure (2) shows the five collinear libration points for the SunEarth-Moon system.

\section{Poincare Surface of Section}

To obtain regular trajectories, the Poincare surface section technique is used.

To verify this, the order of the differential equations of motion are reduced to less order, then integrated numerically through the $(x, y, z)$ variables using the Explicit Runge - Kutta method, with initial conditions, are chosen along the $x$-axis. In the resulting threedimensional space the values of $x$ and $\dot{x}$ can be plotted whenever the infinitesimal body has $y=$ 0 , whenever the trajectory intersects the plane in a particular direction while $\dot{y}>0$. By using the Jacobi constant, it is clear that the resulting $3 \mathrm{D}$ space is reduced to a $2 \mathrm{D}$ subspace. The PSS on the $x, \dot{x}$ plane has been constructed, and the PSS contains discrete points of trajectories.
By solving Equation (18) w.r.t. $\dot{x}^{2}$ at $y=0, z=0, \dot{z}=0$ and $\dot{y}>0$, the resulting could be used as the initial values in the model of calculating the PSS. Figures 3 through 7 show the PSS corresponding to each point of the collinear libration points at different values of Jacobi constant. It is clear that the Poincare mirror symmetry about the $y$ axis is obtained in all cases.

\section{Motion in the Vicinity of the collinear Points}

The Sun-Earth-Moon system is considered, then the motion around collinear libration points is obtained with initial conditions of small displacement from $x_{0}=y_{0}=10^{-4}$ and $\dot{x}_{0}=\dot{y}_{0}=0$, to obtain the family of periodic orbits about each of the collinear libration points the following algorithm is used

1- The values of potentials $\mathrm{U}_{\mathrm{xx}}, \mathrm{U}_{\mathrm{yy}}$ are determined for the corresponding coordinate of libration point.

2- The eigenvalues are obtained from the characteristic equation.

3 - The eigenvalues will be four values ( 2 reals, 2 imaginaries).

4- The two imaginary values responding to give the stable periodic orbits about the libration point.

Now, the linear equations for the motion about collinear libration points of the four-body are written as [18]

$$
\begin{aligned}
& \dot{p}_{x}-2 p_{y}=x U_{x x}+y U_{x y}+z U_{x z}(20.1) \\
& \dot{p}_{y}+2 p_{x}=x U_{x y}+y U_{y y}+z U_{y z} \\
& \dot{p}_{z}=z U_{z z}
\end{aligned}
$$

where the partial derivatives of the effective potential of the four- body problem are obtained as 
Then, the characteristic equation can be rewritten as

$$
\lambda^{4}+\left(4-U_{x x}-U_{y y}\right) \lambda^{2}+U_{x x} U_{y y}=0
$$

At the libration point under study it is easy to obtain the roots of Equation (22), which are the Eigen values $\lambda_{i}, i=1,2,3,4$, then the coordinates $\mathrm{x}$ and $\mathrm{y}$ can be represented as a function of time as

$$
\begin{aligned}
& x=A_{i} \sum_{i=1}^{4} e^{\lambda_{i} t} \\
& y=B_{i} \sum_{i=1}^{4} e^{\lambda_{i} t}
\end{aligned}
$$

where $A_{i}$ and $B_{i}$ are constants and depend on the eigenvalues at each point and the derivatives of the potential, also they are related to each other [19].

$$
B_{i}=\frac{\lambda_{i}^{2}-U_{x x}}{2 \lambda_{i}-U_{x y}} A_{i}=c_{i} A_{i}
$$

\section{RESULTS AND DISCUSSION}

To apply our work the system Sun -Earth Moon - Spacecraft is considered. The canonical units of masses and distances are used in which mass of the Earth

$\mu_{\mathrm{E}}=1-\mu=\frac{m_{1}}{m_{1}+m_{2}}=0.9878715$, mass of the
Moon $\mu_{\mathrm{M}}=\mu=\frac{m_{2}}{m_{1}+m_{2}}=0.0121506683$; .

Mass of the Sun ms $=\frac{m_{s}}{m_{1}+m_{2}}=328900.48$.

The distance between the Sun and the center of the system $=\mathrm{Rs}=389.1723985$.

A code is constructed using MATHEMATICA language to obtain the location of the libration points, Poincare surface sections, and the families of periodic orbits with their eccentricities and their periods. The results obtained as follows:

\subsection{The positions of collinear libration points and Jacobi constant}

By solving Eq. (16) the positions of the five collinear libration points are obtained and from Eq. (19.1) as shown in table (1). Fig.2 illustrates the collinear libration points.

\subsection{The positions of non-linear libration points}

By solving Eqs. (17) With different values of $\theta$ the positions of the non-linear libration points are obtained as shown in table 2 which displays the dimensionless distances for the three non-collinear libration points (L6, L7, and L8) for the Sun-Earth-Moon system with the different values of $\theta$.

Table 1: The five collinear libration points for Sun-Earth-Moon system and its Jacobi constant.

\begin{tabular}{|c|c|c|c|}
\hline Libration points & Dimensionless $\boldsymbol{X}_{\boldsymbol{L}_{\tilde{\boldsymbol{i}}}}$ & $\boldsymbol{X}_{\boldsymbol{L}_{\boldsymbol{i}}}(\mathbf{k m})$ & Jacobi constant C \\
\hline L1 & -1.86027 & -277180 & 7.853603 \\
\hline L2 & -0.897262 & -133691 & 4.098169 \\
\hline L3 & 0.593443 & 884228 & 2.493536 \\
\hline L4 & 0.908389 & 135349 & 4.405134 \\
\hline L5 & 1.05927 & 157830 & 8.827686 \\
\hline
\end{tabular}

Table 2 the three non collinear libration points for Sun-Earth-Moon system.

\begin{tabular}{|c|c|c|c|c|c|c||}
\hline \hline$\theta$ & \multicolumn{2}{|c|}{$\begin{array}{c}\text { L6 } \\
(\mathrm{x}, \mathrm{y})\end{array}$} & \multicolumn{2}{c|}{$\begin{array}{c}2 \\
\text { L8, })\end{array}$} \\
\hline 30 & -0.3591 & 2.3018 & 1.0007 & -0.0730 & 0.07946 & -0.5902 \\
\hline 60 & 2.2203 & 0.7117 & 0.9195 & -0.0196 & -0.5798 & -0.1809 \\
\hline 90 & -2.0827 & 1.0434 & 0.9520 & 0.0642 & -0.2809 & 0.5325 \\
\hline 120 & -1.8971 & -1.3533 & 0.4772 & 0.3412 & 1.048 & 0.0394 \\
\hline 150 & -1.6291 & 1.6662 & 0.4084 & -0.4241 & 1.0407 & -0.0494 \\
\hline 180 & 1.3937 & 1.8668 & 0.9411 & -0.0560 & -0.3703 & -0.4768 \\
\hline
\end{tabular}




\subsection{The Poincare sections}

The steps of section 7 are applied. The numerical solution of the system of Eqs. (13) taken into account the positions of $\mathrm{Li}$ as initial values, will enable to obtain the Poincare sections. Figs. 3, 4, 5, 6, and 7 illustrate the Poincare sections about L1, L2, L3, L4, and L5 respectively.

\subsection{The Exterior Point L1}

To study the motion about each of the libration points L1 is chosen as example in details, then the same is done for all the libration points.

The coordinate of the point L1 $(-1.86027,0)$, and the values of derivatives of potentials $U_{x x}, U_{y y}$ are obtained from Eqs. (21.1) and (21.2), where $U_{x x}=1.32449, U_{y y}=-0.8378$, and by solving Eq.(22) the roots are obtained as $\lambda_{1}=0.54002, \quad \lambda_{2}=-0.54002$, $\lambda_{3}=1.950612 i, \lambda_{4}=-1.950612 i$.

Therefore, the solutions for small displacement near L1 become

$\begin{aligned} x(t)= & 2.47371 * 10^{-6} e^{0.54002 t}+1.0047 * 10^{-5} e^{-0.54002 t}-2.52133 * \\ & 10^{-6} \operatorname{Cos}[1.950612 t]+2.09682 * 10^{-6} \operatorname{Sin}[1.950612 t]\end{aligned}$

$y(t)=-2.36565 * 10^{-6} e^{0.54002 t}+9.60872 * 10^{-6} e^{-0.54002 t}+2.75693 *$ $10^{-6} \operatorname{Cos}[1.950612 t]+3.31508 * 10^{-6} \operatorname{Sin}[1.950612 t]$

Equations (25) represent the trajectories of a spacecraft around the libration point L1 with small displacement from L1. The first two terms of equations (25.1) and (25.2) will make the track to increase with time, so that, to obtain the periodic part of these trajectories, it is more convenient to consider the only terms which include sinusoidal terms. Figure 8 shows that the motion around L1 is periodic motion, its track is an ellipse, and its center lies at L1. Its eccentricity and the period of the orbit are obtained by [19]

$e^{2}=\frac{d^{2}-1}{d^{2}}$

$T=\frac{2 \pi}{s}$

where, $\mathrm{d}=\frac{\lambda_{i}^{2}-U_{x x}}{2 \lambda_{i}-U_{x y}} \lambda_{i}=\lambda_{3,4}$ and $\mathrm{s}$ is coefficient of imaginary parts of $\lambda_{i}$

Then $\mathrm{e}=0.787$ and its synodic period is $\mathrm{T}=3.22113$ unit of time. In the same manner, the periodic orbits about L2, L4 and L5 are shown in figs 9,12 , and 13 respectively and the eccentricities and the periodic orbits are obtained. While fig. 10 shows that the periodic orbit about L3 is not closed for one period, so that fig. 11 is presented to illustrate that the Lissajous orbits are obtained in these case.

\section{CONCLUSION}

In this study, the Hamiltonian system of four-body problem is constructed. The canonical Hamiltonian form is used to obtain the collinear libration points in the system SunEarth-Moon-Spacecraft which the five collinear libration points are explored. The three noncollinear libration points are found for different position with respect to the sun, it is clear that the position of the Sun has an important rule to specify the location of the non-collinear libration points Table (2). The motion about the collinear libration points is studied and gives stability for the specified time. It is found that the longest period of stability is obtained at L2

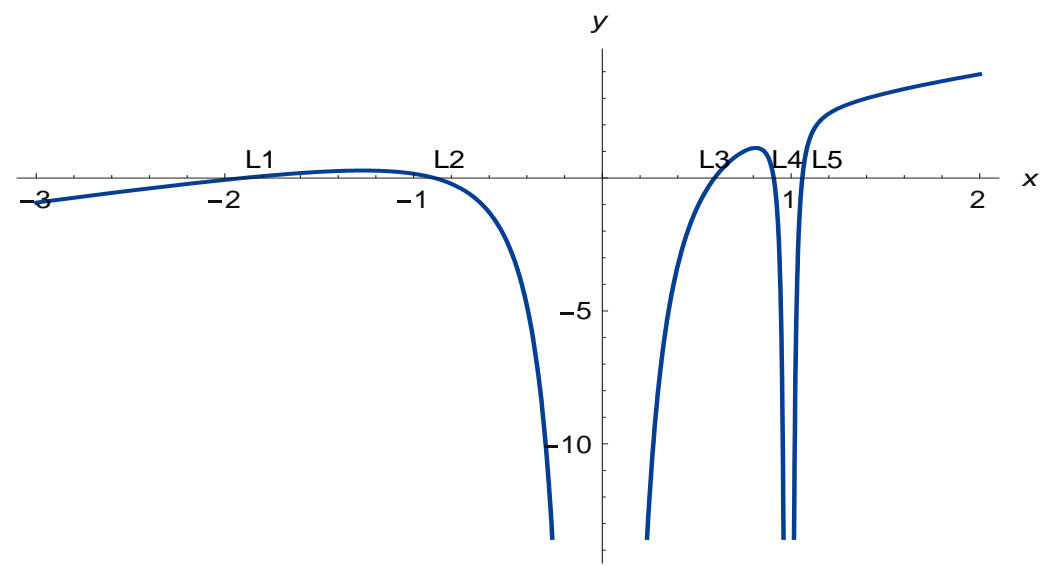

Figure 2: Five collinear points for sun- earth -moon system 
ISMAIL. M.N., et al.,

$\mathrm{T}=4.9573$ unit of time, that means this point is more suitable for the space mission. The Poincare map shows the mirror symmetry which enables the space missions to be more utilized. At L3, some Lissajous orbits are found which can be used for missions which forward outer planets. This study has an important role in the field of space dynamics. The future work in this fields needs to take into account the effects of oblateness of the primaries, the coefficients of the solar sail absorption, and reflection, and the incident angle of the solar radiation pressure.

\section{ACKNOWLEDGMENT}

The authors would like to thank all the team works in this work.

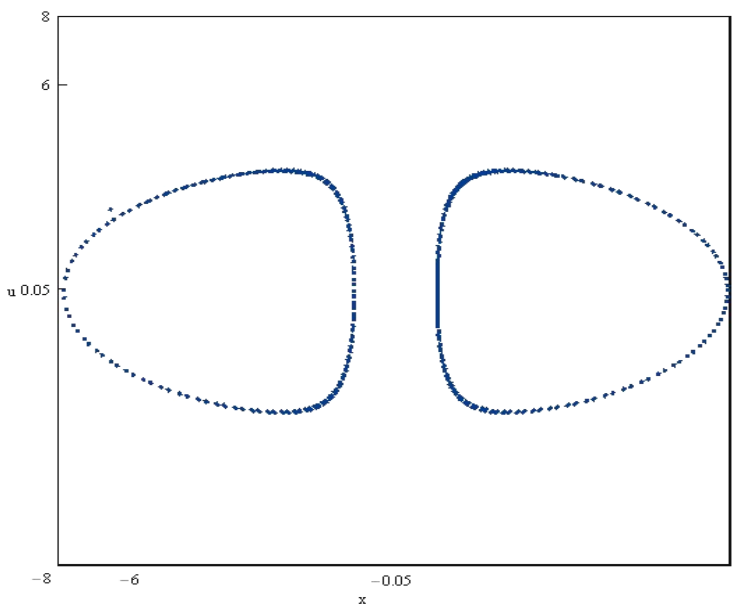

Figure 3: Poincare section at $\mathrm{L} 1=-\mathbf{1 . 8 6}$

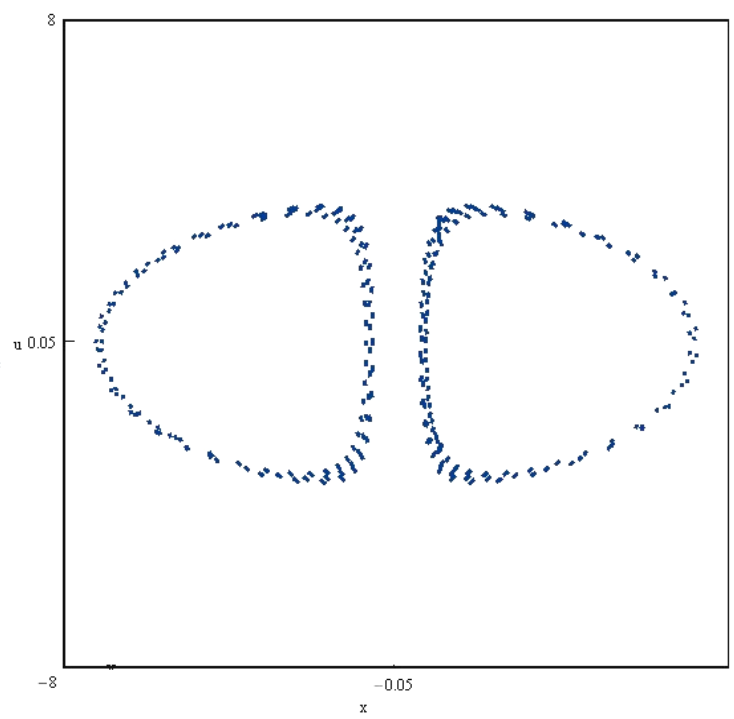

Figure 4: Poincare section at $\mathrm{L} 2=-\mathbf{- 8 6 7 2}$

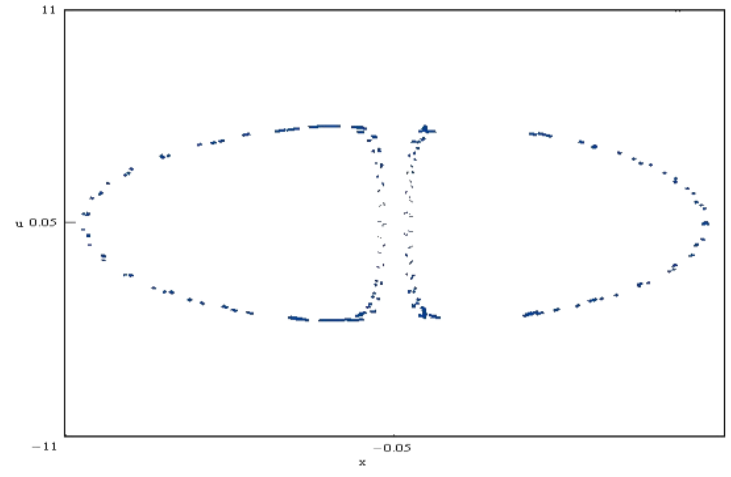

Figure 5: Poincare section At L3=0.59344

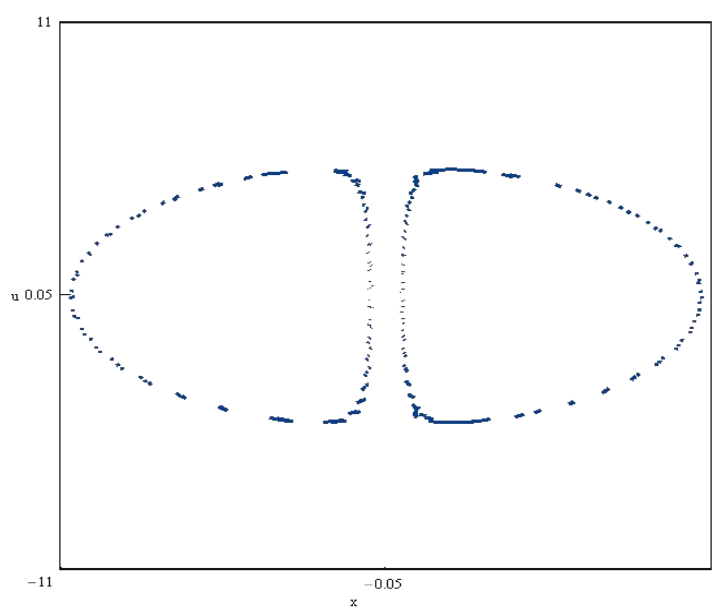

Figure 6: Poincare section at L4 $=0.90838$

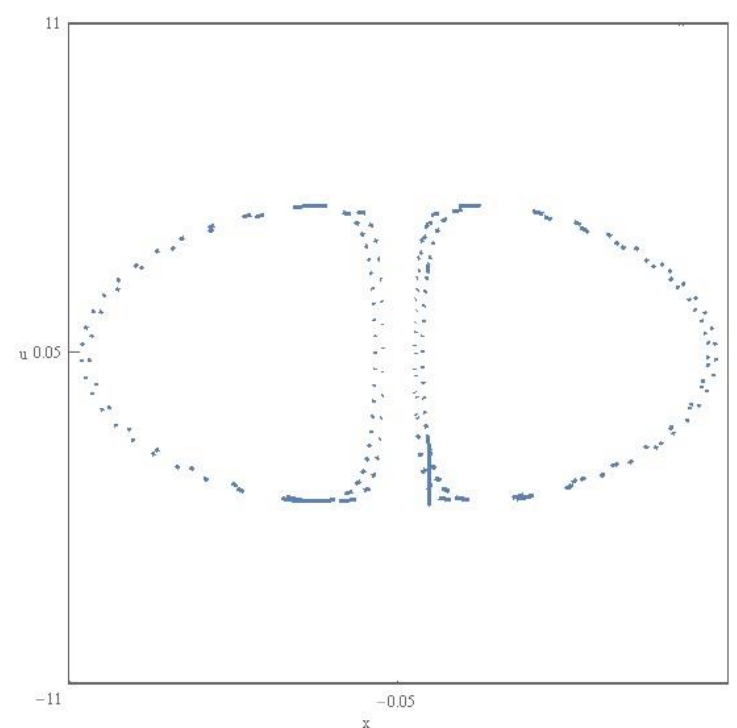

Figure 7: Poincare section at L5=1.024 


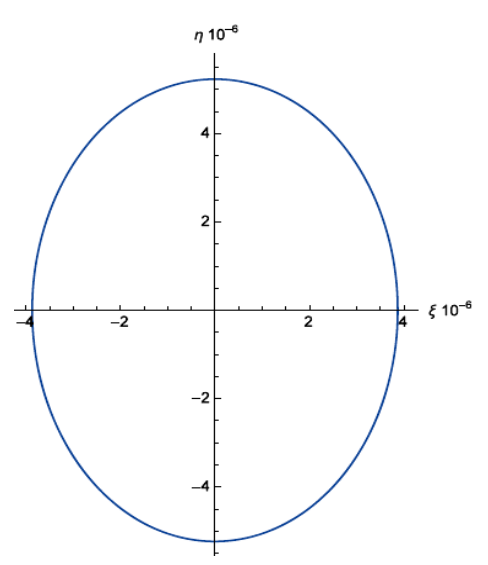

Figure 8: The trajectory around L1 with $e=0.787$. and $T=3.22113$.

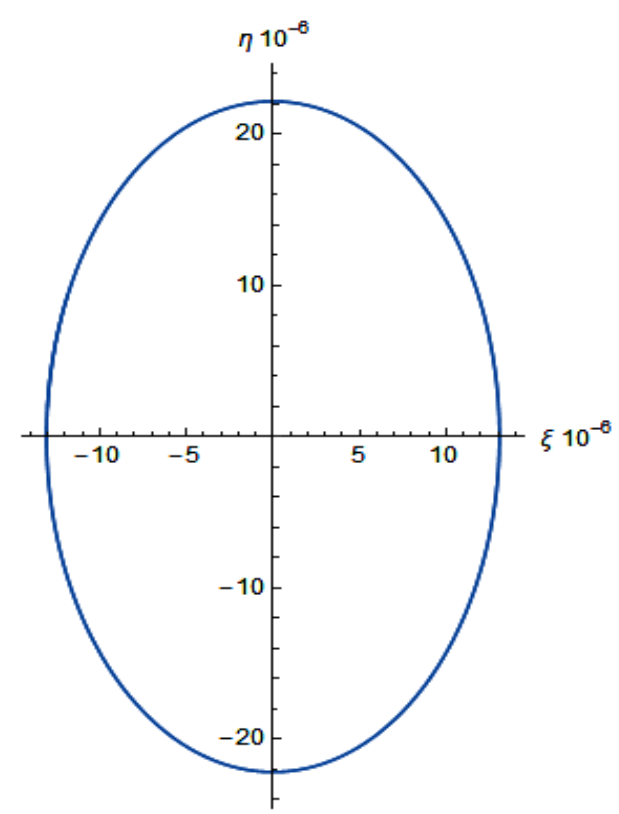

Figure 9: The trajectory around $L 2$ with $e=0.8428$. and $T=4.9573$.

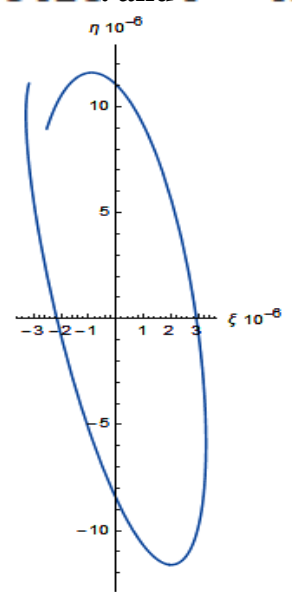

Figure 10: The trajectory around $\mathrm{L3}$ with $e=0.9893$ and $T=2.8242$.

L3 for one period

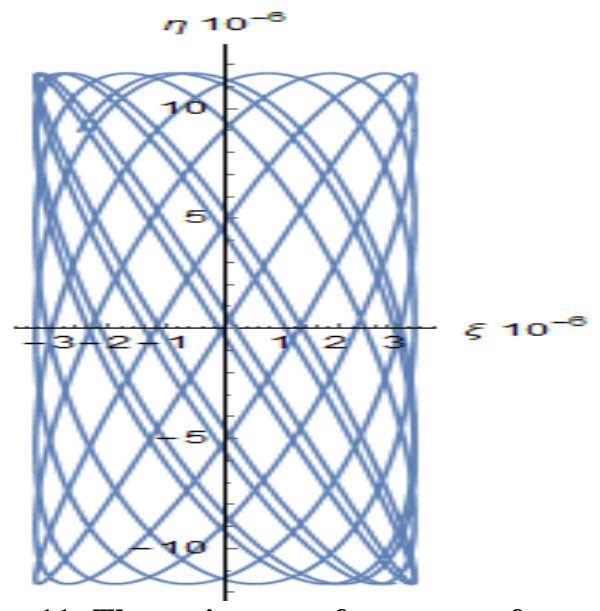

Figure 11: The trajectory of a spacecraft around the point $L 3$ for ten periods.

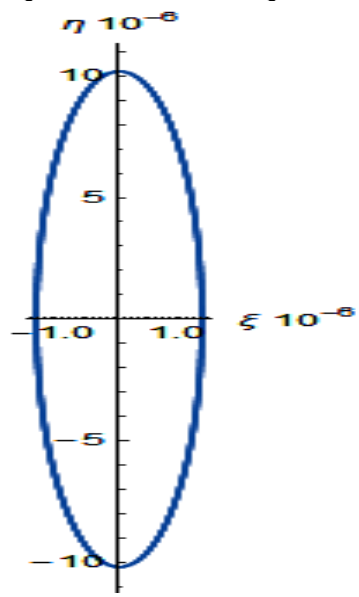

Figure 12: The trajectory around $\mathrm{L} 4$ with $e=0.99989$. and $T=1.23731$.

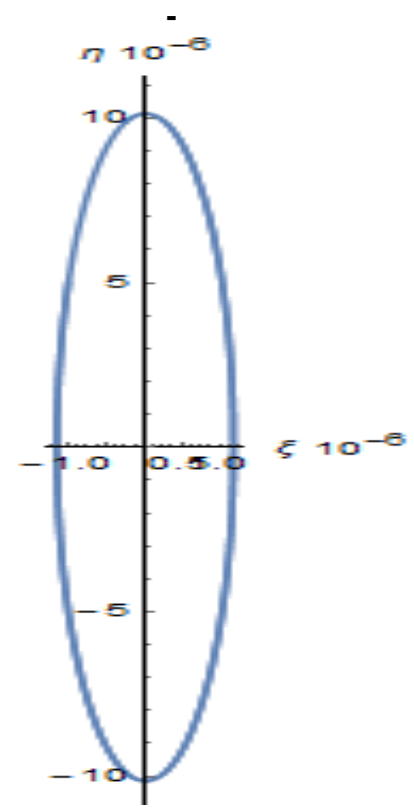

Figure 13: The trajectory around L5 with $e=0.999954$. and $T=1.0701$. 


\section{REFERENCES}

[1] Gabern, F., \& Jorba, A. 2001,“A restricted fourbody model for the dynamics near the Lagrangian points of the Sun-Jupiter system", Discrete and Continuous Dynamical Systems series B, 1(2), 143-182.

[2] Gómez, G., Llibre, J., Martínez, R., \&Simó, C1987," Study on orbits near the triangular libration points in the perturbed restricted three body problem", Final Report Fundacio Empresai Ciencia, Barcelona (Spain).

[3] Jorba, A.2000,"A numerical study on the existence of stable motions near the triangular points of the real Earth-Moon system, A dynamical systems approach to the existence of Trojan motions", Astronomy and Astrophysics, 364, 327-338.

[4] Bell, J,1991," The impact of solar radiation pressure on Sun-Earth L1 libration point orbits", M. S. Thesis, Purdue University, West Lafayette, Indiana, August

[5] Guzman,J 2001,“Spacecraft Trajectory Design in The Context A Coherent Restricted Four-Body Problem", Ph.D. Dissertation Purdue University.

[6] Pernicka, H, J 1990,'The Numerical Determination of Nominal Libration Point Trajectories and Development of A StationKeeping Strategy", Ph.D. Dissertation Purdue University.

[7] Andreu, M, A 1998,"The quasi-bicircular problem", PhD Dissertation, University of Barcelona.

[8] Scheeres, D. 1998," The restricted Hill fourbody problem with applications to the EarthMoon-Sun system. Celestial Mechanics and Dynamical Astronomy, 70(2), 75-98.

[9] Baltagiannis, A. N., \& Papadakis, K. E 2011," Equilibrium points and their stability in the restricted four-body problem", International Journal of Bifurcation and Chaos, 21(08), 21792193.

[10] Baltagiannis, A. N., \& Papadakis, K. E.2011,"Families of periodic orbits in the restricted four-body problem", Astrophysics and Space Science, 336(2), 357-367.

[11] Burgos-Garcia, J 2013,"Regularization in the Restricted Four Body Problem", arXiv preprint arXiv:1310.5274.

[12] Burgos-García, J., \& Delgado, J 2013,"Periodic orbits in the restricted four-body problem with two equal masses", Astrophysics and Space Science, 345(2), 247-263.

[13] Schechter, H. B 1968."Three-dimensional nonlinear stability analysis of the sun-perturbed earth-moon equilateral points", AIAA Journal, 6(7), 1223-1228

[14] Carpenter, L., \& Kolenkiewicz, R 1968,"Stable periodic orbits about the sun perturbed earthmoon triangular points", AIAA Journal, 6(7), 1301-1304.

[15] Dutt, P., \& Sharma, R. K 2010,"Analysis of periodic and quasi-periodic orbits in the EarthMoon system", Journal of guidance, control, and dynamics, 33(3), 1010-1017.

[16] Dutt, P., \& Sharma, R. K2012,“On the evolution of the ' $\mathrm{f}$ ' family in the restricted three-body problem. Astrophysics and Space Science, 340(1), 63-70.

[17] Ibrahim, A.H., Ismail, M.N., Zaghrout, A.S., Younis, S.H. and El Shikh, M.O 2018,"Lissajous Orbits at the Collinear Libration Points in the Restricted Three-Body Problem with Ob-lateness", World Journal of Mechanics, 8, 242-252.

[18] Ibrahim, A. H 2017,'”Effect of Solar Radiation Pressure onto the Motion of Many Body Problem", Ph.D. Thesis, Al-Azhar University, Cairo.

[19] Ibrahim, A. H., Ismail, M. N., Zaghrout, A. S., Younis, S. H., \&Shikh, M. O 2018,“Orbital Motion Around the Collinear Libration Points of the Restricted Three-Body Problem", Journal of Advances in Mathematics and Computer Science, 29(1), 1-16. https:// doi.org/ 10.9734/JAMCS/2018/43370.

[20] Szebehley, V. (1967),"Theory of orbits. The Restricted Problem of Three Bodies", Academic press.

[21] Reena Kumari and Badam Singh Kushvah (2013):" Equilibrium points and zero velocity surfaces in the restricted four-body problem with solar wind drag", Astrophysics Space Science 344:347-359. DOI 10.1007/s10509012-1340-y 


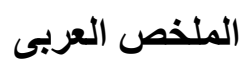

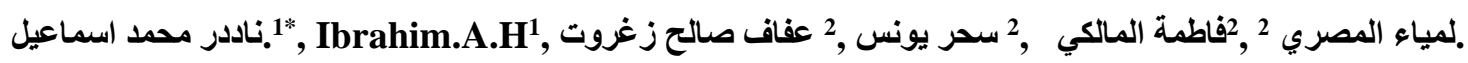

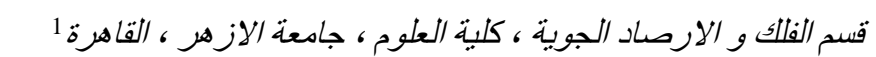

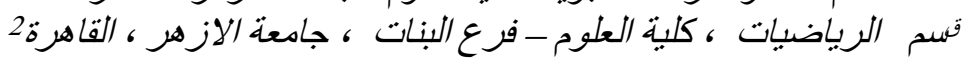

في هذا العمل ، صيغت مشكلة الأجسام الأربعة المقيدة في شكل هاملتون. يتم الحصول على الثكل الكنسي للنظام الذي يمثل معادلات الحركة. يتم الحصول على نقاط الاتزان الخطية وقد وجدنا أن هناك خمس نقاط اتز ان خطية. نم العثور على نقاط الاتزان غير الخطية وهي ثنات نقاط اتزان غير خطية ، وتم الحصول عليها لزوايا مختلفة بين خط اتجاه الثمس ومستوى الأرض- القمر. تم دراسة المدارات الدورية حول كل نقطة من نقاط الاتزان الخطية هذه باستخدام طريقتين. تعتمد الطريقة الأولى على تقليل درجة المعادلات التفاضلية

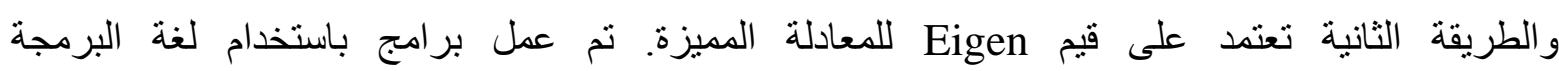
MATHEMATICA الفضائية. تم الحصول على أشكال Poincare باستخدام الطريقة الأولى ، حيث ينم استخدام هذه الأشكال لتوضيح نقاط التقاطع في المسار ات مع المستوى العمودي لمستوى الحركة حول كل نقطة من نقاط الاتزان الخطية. تم الحصول على التمانل المرآة حول كل من هذه النقاط. النتائج التي تم الحصول عليها عن طريق الطريقة الثانية هي مدارات Lyapunov، والمدارات Lissajous حول كل نقطة من نقاط الاتزان الخطية. تم الحصول على المدارات الدورية وفترات كل مدار ـ توضح هذه الدراسة أن الحركة حول نقطة الاتزان الثانية

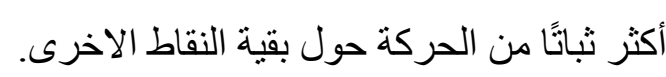

